Ana Marlúcia Oliveira Assis 1 Leonor Maria Pacheco Santos 1 Matildes da Silva Prado 1 Maísa Cruz Martins 2 Maurício Lima Barreto 3

\section{Tolerância à aplicação de megadoses de vitamina $A$ associada à vacinação em crianças no Nordeste do Brasil}

\author{
Tolerance of vitamin A application \\ associated with mass immunization \\ of children in Northeast Brazil
}

\footnotetext{
1 Escola de Nutrição, Universidade Federal da Bahia. Rua Araújo Pinho 32, Canela, Salvador, BA 40110-170, Brasil. amos@ufba.br 2 Secretaria de Saúde do Estado da Bahia. Centro Administrativo da Bahia, Salvador, BA 41746-900, Brasil.

3 Instituto de Saúde Coletiva, Universidade Federal da Bahia. Rua Padre Feijó 29, 4o andar, Canela, Salvador, BA 40110-170, Brasil.
}

Abstract A follow-up study was carried out in two localities in the semi-arid region of the State of Bahia, Northeast Brazil, with the aim of identifying the occurrence and nature of possible acute si de effects subsequent to vitamin A megadose supplement given together with mass immunization in children 6-59 months old. The sample consisted of 852 children, 416 from the county of Teofilandia who recei ved vitamin A together with vaccines and 436 from Santa Barbara, who received only vaccine. In the 24 hours before immunization, children from both groups had similar incidences of diarrhea, fever, and vomiting. Anorexia was more preval ent in Teofilandia and remained so throughout the study period. The results suggest that acute side effects like diarrhea, vomiting, fever, or anorexia were not associated with the vitamin A dosage given with mass OPV, DPT, and measles immunization.

Key words Vitamin A; Supplementary Feeding; Immunization Program

Resumo Um estudo de seguimento foi desenvolvido em duas locali dades do semi-ári do do estado da Bahia, Nordeste do Brasil, com o objetivo de i dentificar a ocorrência ea natureza de possíveis efeitos adversos agudos em conseqüência da suplementação com megadoses de vitamina $A$ (100.000 e 200.000 UI) ofereci da junto com imunização em massa, a crianças de seis a 59 meses de idade. A amostra do estudo foi composta por 852 crianças; 416 do município de Teofilândia integraram o grupo que recebeu a vitamina A com as vacinas e 436 crianças de Santa Bárbara foram incluídas no grupo que recebeu somentea vacina. Nas 24 horas que antecederam a vacinação, as crianças dos dois grupos referiram similar freqü ência de diarréia, febre e vômi to; a anorexia foi mais prevalente em Teofilândia e persistiu durante todo o período de seguimento. Os resultados sugerem que nenhum efei to adverso agudo, em especial diarréia, vômito, febre ou anorexia, esteve associado à ingestão da vitamina A combinada à vacinação em massa, particularmente à Sabin, DPT e anti-sarampo.

Palavras-chave Vitamina A; Suplementação Alimentar; Programas deVacinação 
Introdução

A deficiência de vitamina $A$ é uma das importantes deficiências nutricionais no mundo subdesenvolvido (FAO, 1992). No Brasil, até o momento, os estados do Nordeste têm registrado sua ocorrência, principalmente em crianças pré-escolares (Prado et al., 1995; Santos et al., 1996).

A deficiência desse micronutriente está positivamente associada à morbimortalidade em pré-escolares. A suplementação de vitamina A oferecida a esse grupo populacional diminui a mortalidade por todas as causas na ordem de 23\% a 34\%, conforme estimativas de meta-análises (Tonascia, 1992; Beaton et al., 1993; Fawzi et al., 1993; Glasziou \& MacKerras, 1993), e também a morbidade, através da diminuição da severidade de enfermidades infecciosas, em especial a diarréia (Barreto et al., 1994; Bhandari et al., 1994).

Após os resultados dos estudos de intervenção com vitamina A, que comprovaram a relação de causa e efeito entre a suplementação com esse micronutriente e a redução na taxa da mortalidade na infância e a severidade das doenças infecciosas, o Fundo das Nações Unidas para a Infância (UNICEF), a Organização Mundial de Saúde (OMS) e a Organização das Nações Unidas para a Agricultura e a Alimentação (FAO) têm preconizado a necessidade de instituir programas de prevenção da deficiência desse micronutriente. A proposta de "eliminação virtual da deficiência de vitamina A e suas conseqüências" foi referendada na Conferência Internacional de Nutrição, em 1992, e passou a constituir, também, objeto de compromisso de chefes de Estado de países onde a deficiência desse micronutriente é endêmica, inclusive o Brasil (FAO, 1992). Essa proposta inclui ações a longo prazo, que se sustentam nos programas de diversificação dietética e de saúde pública, e ações a curto prazo, representadas, especialmente, pelo programa de suplementação em massa com a vitamina A para as crianças menores de cinco anos de idade sujeitas aos riscos da deficiência. A distribuição da vitamina A integrada ao programa de imunização é um dos mecanismos propostos pela OMS e pelo International Vitamin A Consultative Group (IVACG) para tornar eficiente o combate à deficiência da vitamina A (WHO/IVACG, 1993).

Alguns estudos têm detectado que a diarréia (Hathcock et al., 1990), náuseas, dor de cabeça, vômito (Florentino et al., 1990) e abaulamento de fontanela em crianças (Agoestina et al., 1994) constituem efeitos adversos agudos associados à ingestão de altas ou megadoses de vitamina A. Outras investigações não detectaram efeitos tóxicos agudos, tais como dor de cabeça e náuseas (Batista et al., 1974) ou diarréia e febre (West et al., 1992) face à suplementação. No entanto, esses estudos não associaram a suplementação da vitamina $\mathrm{A}$ com vacinas, desconhecendo-se, assim, a existência de um efeito sinérgico entre a vitamina $A$ e os componentes das vacinas, tampouco a potencialização desses efeitos, e, ainda, se estes efeitos são semelhantes para aquelas crianças que estão tomando a primeira dose ou as doses subsequentes da vacina.

No Brasil, a partir de 1983, o Ministério da Saúde, através do Instituto Nacional de Alimentação e Nutrição (INAN), distribuiu cápsulas de vitamina $A$ associadas à vacina antipólio para pré-escolares. No entanto, na ocasião, não foram avaliados os possíveis efeitos adversos dessa intervenção. Através da Portaria no 2.160, de 29 de dezembro de 1994, do Ministério da Saúde, foi instituído o Programa de Combate à Deficiência de Vitamina A (Brasil, 1994) e, a partir de então, a distribuição de megadoses a pré-escolares intensificou-se.

A suplementação em massa de vitamina A em regiões onde a deficiência é endêmica favorece a redução da taxa de mortalidade em crianças menores de cinco anos de idade (Beaton et al., 1993) e representa, também, a possibilidade de reduzir a severidade das doenças diarréicas (Barreto et al., 1994). Considerando o efeito protetor que a suplementação desse micronutriente exerce sobre a saú de infantil, esforços devem ser conduzidos no sentido de elucidar a relação entre doses maciças de vitamina A e efeitos indesejáveis em crianças, em especial quando a vitamina A é oferecida em conjunto com a vacina.

Assim, o objetivo deste estudo foi identificar a ocorrência e a natureza de possíveis efeitos adversos agudos como conseqüência da suplementação com vitamina A oferecida junto com vacinas em campanhas de imunização em massa, em crianças de seis a 59 meses de idade.

\section{População e método}

Este estudo foi desenvolvido em duas cidades da região Nordeste do Brasil, localizadas no semi-árido do Estado da Bahia, durante a campanha de vacinação em massa em outubro de 1995. Para essa região, tem sido documentado que a prevalência de baixos níveis de retinol sérico em crianças é elevada (Prado et al., 1995; 
Santos et al., 1996). Em uma das cidades, Teofilândia, foi oferecida a suplementação de vitamina A, junto com as vacinas do Programa Nacional de Imunização (grupo intervenção), enquanto na cidade de Santa Bárbara foram oferecidas somente as vacinas (grupo controle). As crianças de seis a 12 meses e as maiores de um ano de idade receberam, respectivamente, 100.000 e 200.000 UI de vitamina A oleosa, na forma de xarope. Ao final do seguimento, foi distribuída a vitamina $A$ às crianças da cidade que funcionou como controle nesse estudo.

A distância entre as duas cidades é de aproximadamente $40 \mathrm{~km}$. Segundo o censo demográfico de 1991, os menores de cinco anos de idade totalizavam 651 em Teofilândia e 578 em Santa Bárbara. Os chefes de família tinham rendimento médio de até um salário mínimo em $37,8 \%$ dos casos no município de Teofilândia e, em Santa Bárbara, esse percentual era de $55,6 \%$ (IBGE, 1991).

\section{Tipo de estudo}

Este é um estudo de intervenção desenvolvido em duas comunidades. Em uma comunidade a campanha de vacinação ocorreu normalmente (comunidade controle) e na outra à imunização ministrada associou-se uma megadose de vitamina A (comunidade intervenção). A adoção desse modelo de investigação ocorreu em virtude da necessidade de adequar os objetivos do estudo à estrutura operacional de um programa governamental rotineiro de vacinação ao qual se associa uma intervenção.

\section{A mostra}

Através de um censo realizado nas duas cidades, no dia anterior à vacinação, foram identificadas 884 crianças elegíveis para o estudo. Contudo, 13 deixaram de comparecer à vacinação, totalizando, assim, 871 crianças de seis a 59 meses de idade que procuraram os postos de vacinação (421 crianças em Teofilândia e 450 em Santa Bárbara). A perda no seguimento foi de nove crianças (quatro no município intervenção e cinco no município controle): cinco por não ter comprovada a idade e quatro por motivo de viagem. Considerando que somente dez crianças tomaram BCG nos dois municípios, decidiu-se também exclui-las da amostra. Assim, os resultados apresentados são relativos a 852 crianças: 416 integraram o grupo que recebeu a vitamina $A$ em conjunto com as vacinas e 436 foram incluídas no grupo que recebeu somente a imunização. Na ocasião, foram distribuídas 1.029 doses de vacinas de interesse para este estudo: 845 doses de antipólio oral (49,1\% em Teofilândia e 50,9\% em Santa Bárbara, $p=0,12), 121$ doses de DPT (38,8\% em Teofilândia e 61,2\% em Santa Bárbara, $p=0,01$ ) e 63 doses de anti-sarampo (66,7\% em Teofilândia e 33,3\% em Santa Bárbara, $p=0,003$ ).

\section{Coleta de dados}

No censo realizado no dia anterior à vacinação foram identificadas as crianças potenciais integrantes da amostra dessa investigação. Após a informação sobre os objetivos estudo, obteve-se o consentimento informado da mãe ou responsável pela criança para a participação no estudo. Na oportunidade, as mães ou responsáveis foram inquiridos sobre o estado de saúde de sua(s) criança(s), em especial sobre a ocorrência de diarréia, febre, vômito e anorexia, nas 24 horas que precederam a distribuição da vitamina A e da vacinação. Destacou-se, como objetivo do estudo, conhecer os principais sinais, sintomas e queixas que as crianças viessem a apresentar depois das vacinas. Nenhum aspecto relacionado aos possíveis efeitos da suplementação de vitamina A foi referido para evitar que as mães ou responsáveis fossem sugestionados sobre alguns dos sintomas pesquisados. Na ocasião, destacou-se a importância da vacinação como uma forma eficaz no combate à morbimortalidade infantil, e a mãe ou o responsável foram, mais uma vez, convidados a comparecer, no dia seguinte, a um posto de vacinação próximo de sua residência com a(s) sua(s) criança(s). Algumas questões referentes à situação sócio-econômica foram também incluídas no questionário.

As visitas para coleta de informação foram repetidas 24,48 e 72 horas após a vacinação, sempre nos mesmos horários ou os mais próximos possíveis. As informações sobre o estado de saúde das crianças foram referidos pela mãe ou responsável. Os dados foram coletados por alunas da Escola de Nutrição da Universidade Federal da Bahia e por professoras primárias da região, devidamente treinadas. A coleta da informação foi supervisionada pelos investigadores principais. Os questionários foram revisados em campo pelos supervisores e, quando indicado, as informações foram coletadas outra vez. Dez por cento dos questionários foram revisados em campo pelos pesquisadores principais. 
A nálise

As análises foram realizadas contemplando os grupos, independentemente do tipo de vacina (antipólio oral, DPT e anti-sarampo), e, ainda, as crianças que tomaram exclusivamente a vacina antipólio oral. Calculou-se a prevalência dos sinais e sintomas investigados nas 24,48 e 72 horas após a vacinação.

O teste de qui-quadrado foi utilizado para comparar os grupos entre si e para testar as hipóteses de interesse. Quando indicado, utilizou-se a análise estratificada para avaliar os possíveis efeitos de fatores confundidores. $\mathrm{O}$ teste exato de Fisher foi adotado quando apropriado. Aceitou-se um nível de significância de $p<0,05$ e testes bicaudais para comprovação das hipóteses.

\section{Resultados}

A distribuição das variáveis biológicas (idade e sexo) foi similar entre os grupos do estudo. A escolaridade materna e a existência de sanitário ligado a rede de esgotamento sanitário distribuíram-se de maneira diferenciada entre os grupos, indicando uma situação mais privilegiada para as famílias das crianças de Teofilândia (grupo suplementado). Assim, todas as análises aqui apresentadas foram controladas para o possível efeito confundidor dessas duas variáveis. No dia que precedeu a vacinação/suplementação, nenhuma diferença estatisticamente significante foi observada para as prevalências de febre, diarréia e vômito entre as crianças dos dois municípios. No entanto, observou-se entre as crianças do município de Teofilândia prevalência mais elevada de anorexia $(p=0,00)$ (Tabela 1$)$.

Os resultados das análises referentes à prevalência diária dos sintomas no período do seguimento estão apresentadas na Tabela 2. Nas 24 horas após a administração das vacinas/ suplementação, a prevalência de febre e diarréia foi mais elevada para as crianças que não receberam a vitamina A, destacando-se, inclusive, a significância estatística para a prevalência de febre entre os grupos. No entanto, quando a análise foi controlada para o uso da vacina DPT, esse efeito desaparece. Quando a análise foi realizada considerando somente as crianças que receberam a vitamina $A$ juntamente com a vacina antipólio oral, não são observadas, entre os grupos, diferenças significantes para esse sintoma. A prevalência de vômito, em todo o período do acompanhamento e independente do tipo de vacina oferecida, foi simi-
Iar nos dois grupos. A diferença estatisticamente significante observada para a anorexia antes do oferecimento da vacina continuou para os períodos de 24, 48 e 72 horas após a vacinação para a grupo como um todo e para aquelas crianças que tomaram exclusivamente a vacina anti pólio oral. A análise controlada pela escolaridade materna mostra que essa diferença é mantida somente para a mais alta escolaridade, indicando que as mães com maior nível de instrução referiram com mais freqüência a anorexia de seus filhos do que aquelas sem nenhuma escolaridade. Essa mesma análise realizada segundo a situação do esgotamento sanitário não altera esses resultados.

\section{Discussão}

Os resultados deste estudo indicam que o oferecimento da vitamina $A$ associado ao esquema de imunização em massa não eleva a taxa de efeitos adversos, em especial diarréia, febre, vômito e anorexia, em crianças de seis meses a cinco anos de idade.

Poucos são os estudos que abordam a potencialização do efeito adverso da vitamina A oferecida juntamente com vacinas. Alguns deles têm voltado sua atenção para os efeitos neurológicos. Nesse aspecto, os resultados não são consensuais. O abaulamento de fontanela como uma das implicações neurológicas do oferecimento da vitamina A junto com vacina é registrado por West et al. (1992), DiFrancisco et al. (1993) e Agoestina et al. (1994), embora Stabell et al. (1995) não o identificassem em crianças de seis a nove meses de idade que receberam, respectivamente, 100.000 e $200.000 \mathrm{UI}$ de vitamina $A$ associada à vacina anti-sarampo. É conhecido, já há algumas décadas, que tanto o excesso de vitamina A quanto o componente pertussis da DPT são responsáveis por complicações neurológicas em crianças (Ström, 1967; Jacob, 1979).

A expressão dos efeitos adversos da associação da suplementação com a vacina em outros órgãos e sistemas tem sido abordada em poucos estudos, e tampouco os resultados são consensuais. O primeiro relato da complicação do oferecimento de 200.000 UI de vitamina A em conjunto com a vacina DPT foi realizado por Ray et al. (1984) em uma amostra de 24 crianças menores de cinco anos de idade. Os autores relatam a história de complicação em cinco delas (20,8\%). Em 16,6\% foi observada a ocorrência de vômito, e todas tiveram febre. Os sintomas foram observados vinte horas depois da administração da vitamina A, desaparecen- 
do no período entre 36 e 48 horas. Contudo, a amostra muito pequena e a inexistência de um grupo controle, aliadas à fragilidade da análise estatística desse estudo, não permitem relacionar esses efeitos à associação entre as duas drogas, tornando-o de relevância limitada.

Similarmente aos nossos resultados, DiFrancisco et al. (1993) tampouco identificaram associação entre febre e o oferecimento de vitamina A junto com as vacinas antipólio oral e DPT em crianças de um a três meses e meio de vida, que receberam $15 \mathrm{~g}$ (50.000 UI) de vitamina A na ocasião da vacinação em massa.

Curiosamente, em nosso estudo, a mais elevada prevalência da febre nas 24 horas do seguimento foi identificada nas crianças que não associaram a vacina à vitamina $A$ (Tabela 2). O controle desse resultado pelo uso de vacina DPT sugere que a febre tenha ocorrido em razão de componentes dessa vacina. Alguns estudos já demonstraram que a fração pertussis da vacina DPT está associado a efeitos adversos, tais como febre (Jones et al., 1992) e diarréia (Floyd \& Freeling, 1992).

A mais alta ocorrência de anorexia nas crianças do Município de Teofilândia, observada antes da intervenção, perdurou por todo o período do seguimento, indicando que este efeito não está associado ao uso da vitamina $A$ combinado à vacinação em massa, mas, provavelmente, ao fato de as mães de mais alta escolaridade referirem a anorexia de seus filhos com mais freqüência.

Sobre essa questão, é interessante comentar que o cuidado materno é indispensável para assegurar o desenvolvimento físico, emocional e intelectual da criança. A capacidade materna em dedicar tempo e atenção necessários para o garantir o adequado estado de saúde da criança pode, por sua vez, estar associada ao seu nível de escolaridade. É provável que a instrução materna possa ser considerada uma condição necessária, ainda que não suficiente, para assegurar a saúde da criança (Longhurst \& Tomkins, 1995). Nesse contexto, pode-se compreender que as mães de mais alta escolaridade deste estudo possam ter dedicado mais tempo e atenção às suas crianças e, assim, foram propensas a referirem com mais freqüência a falta de apetite de seus filhos do que as mães de mais baixa ou nenhuma escolaridade. Por outro lado, não se pode deixar de referir que a escassez de alimentos nas classes mais pobres pode funcionar como um fator limitante para que a criança expresse a sua situação de anorexia.

Assim, os nossos resultados sugerem que não há potencialização do efeito adverso da vi- tamina A quando esta é oferecida junto com as vacinas antipólio oral, DPT e anti-sarampo. Apesar de se desconhecer o estado nutricional de vitamina A no início desse estudo, há evidências de que as crianças dessa região apresentam altas prevalências de inadequação dos níveis de retinol sérico. Salienta-se, ainda, que o oferecimento da vitamina A em massa foi realizado pela primeira vez para essa população. Sob essas circunstâncias, a administração concomitante de vitamina $A$ e vacinas não produziu efeitos adversos nesse grupo de crianças.

O pequeno número de crianças que recebeu a vacina DPT e as demais vacinas injetáveis impossibilitou a realização das análises segundo a idade e as doses acumuladas dessa vacina. Assim, não foi possível avaliar os possíveis efeitos adversos quando a vitamina A é oferecida a crianças sujeitas a repeti das imunizações.

Tabela 1

Características biológicas e sócio-econômicas e a distribuição da prevalência da diarréia, febre, vômito e anorexia nas 24 horas que antecederam o acompanhamento, entre as crianças das duas cidades do semi-árido da Bahia, 1995.

\begin{tabular}{|c|c|c|c|c|c|c|}
\hline & \multicolumn{2}{|c|}{$\begin{array}{l}\text { Vacina + } \\
\text { Vitamina A }\end{array}$} & \multicolumn{2}{|c|}{ Vacina } & $\chi^{2}$ & $\mathrm{p}$ \\
\hline \multicolumn{7}{|l|}{ Idade (meses) } \\
\hline 6 a 11 & 40 & 9,6 & 46 & 10,6 & 4,35 & 0,36 \\
\hline 12 a 23 & 79 & 19,0 & 105 & 24,1 & & \\
\hline 24 a 35 & 105 & 25,2 & 93 & 21,3 & & \\
\hline 36 a 47 & 105 & 25,2 & 105 & 24,1 & & \\
\hline 48 a 60 & 87 & 20,9 & 87 & 20,0 & & \\
\hline \multicolumn{7}{|l|}{ Sexo } \\
\hline Masculino & 208 & 50,0 & 215 & 49,3 & 0,04 & 0,84 \\
\hline Feminino & 208 & 50,0 & 221 & 50,7 & & \\
\hline \multicolumn{7}{|l|}{$\begin{array}{l}\text { Escolaridade materna } \\
\text { (em anos de estudo) }\end{array}$} \\
\hline Não estudou & 21 & 5,1 & 33 & 7,9 & 38,7 & 0,00 \\
\hline 1 a 8 & 216 & 52,4 & 290 & 69,7 & & \\
\hline 9 a 11 & 161 & 39,1 & 87 & 20,9 & & \\
\hline+11 & 14 & 3,4 & 6 & 1,4 & & \\
\hline $\begin{array}{l}\text { Domicílio ligado } \\
\text { à rede de esgoto }\end{array}$ & 331 & 79,8 & 291 & 67,8 & 15,47 & 0,00 \\
\hline O corrência de diarréia* & 27 & 6,5 & 31 & 7,1 & 0,12 & 0,71 \\
\hline O corrência de febre* & 14 & 3,4 & 22 & 5,0 & 1,48 & 0,22 \\
\hline O corrência de vômito* & 9 & 2,2 & 8 & 1,8 & 0,11 & 0,73 \\
\hline O corrência de anorexia* & 114 & 27,4 & 65 & 14,9 & 20,02 & 0,00 \\
\hline Em uso de leite materno* & 35 & 8,4 & 32 & 7,3 & 0,33 & 0,56 \\
\hline
\end{tabular}

* nas 24 horas anteriores ao estudo. 
Tabela 2

Prevalência diária de diarréia, febre, vômito e anorexia no período de 24,48 e 72 horas de seguimento entre as crianças do grupo que tomou as vacinas associadas à vitamina $\mathrm{A}$ e aquele que recebeu somente as vacinas.

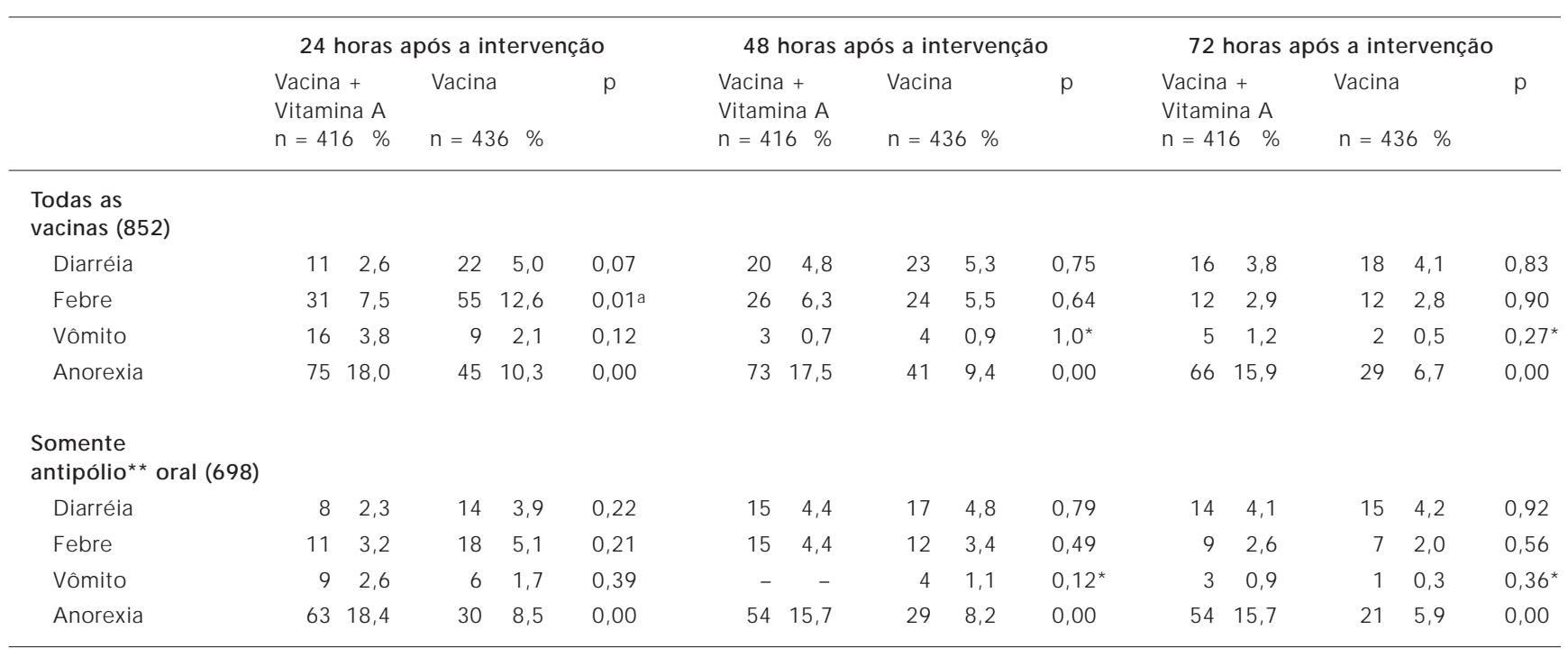

a Quando controlado pelo uso de DPT, o efeito estatisticamente significante observado para a febre nas primeiras 24 horas de seguimento

desaparece ( $p=1$; teste exato de Fisher, bicaudal), e, nos demais períodos, os resultados não são alterados.

* Teste exato de Fisher (bi-caudal).

** $\mathrm{n}=343$ (vitamina A + vacina ); $\mathrm{n}=345$ (vacina ).

\section{Agradecimentos}

Agradecemos às estudantes da Escola de Nutrição da Universidade Federal da Bahia, abaixo relacionadas, pela participação na coleta de dados desta investigação: Adenilda Queiroz dos Santos, Aline Maria Peixoto Lima, Andréa Ferreira da Silva, Carla Cristina Barreto da Silva, Cleide Melo Prates, Daniela Augusta de Castro, Edneuza Alves do Nascimento, Eliacy Góes Souza, Emilia Alves de Santana, Elaine Zanazi de Almeida, Juliana Mota Oliveira dos Santos, Luciara Leite de Brito, Luciana Alaíde Alves Santana, Luciana Ferreira da Silva, Lucivalda Pereira Magalhães, Mabel de Brito Santos, Manuela Lira da Cruz Gouveia, Márcia Maria Pessoa Portugal, Maria Aparecida Anunciação Dias, Maria Consuelo Alvarez Costa, Micheli Dantas Soares, Nêila Ceberg Sodré de Andrade, Patricia Almeida Jacob Netto, Sandra Marciara Lima de Jesus, Sarah Maria Rocha Duarte, Suley Santos Alves, Tatiana Avena de Almeida, Valterlinda Alves de Oliveira e Viviane Sahade Souza.

\section{Referências}

AGOESTINA, T.; HUMPHREY, J. H.; TAYLOR, G. A.; USMAN, A.; SUBARDJA, D.; HIDAYAT, S.; NURACHIM, M.; LEE, W. U.; FRIEDMAN, D. S.; WEST, K. P. \& SOMMER, A., 1994. Safety of one $52 \mathrm{~mol}$ (50.000 IU) oral dose of vitamin A administered to neonates. Bulletin of theWorld Health Organization, 72:859-868.

BARRETO, M. L.; SANTOS, L. M. P.; ASSIS, A. M. O.; ARAÚJO, M. P. N.; FARENZENA, G. G. \& SANTOS, P. A., 1994. Effect of vitamin A supplementation on diarrhea and acute lower-respiratory tract infection in young children in Brazil. Lancet, 334: 228-231.

BATISTA, M.; VARFIA, R. M.; TEIXEIRA, S. G. \& SIMMONS, W. K., 1974. A study in the use of oral massive doses of vitamin A. Ecology of Food and Nutrition, 3:293-298.

BHANDARI, N.; BHAN, M. K. \& SAZAWAL, S., 1994. Impact of massive dose of vitamin $A$ given to preschool with acute diarrhea on subsequent respiratory and diarrhoeal morbidity. BMJ, 309: 1404-1407. 
BRASI L, 1994. Portaria no 2.160, de 23 de dezembro de 1994. Cria, no Instituto Nacional de Alimentação e Nutricão (INAN), o Programa Nacional de Controle das Deficiências de Vitamina A, e dá outras providências. Brasília: Diário Oficial da República Federativa da Brasil, n. 248, p. 21263, 30 dez. Seção 1.

BEATON, G. H.; MARTORELL, R.; L'ABBÉ, K. A.; EDMONSTON, B.; MCCABE, G.; ROSSI, A. C. \& HARVEY, B., 1993. Effectiveness of Vitamin A Supplementation in the Control of Young Child Morbidity and Mortality in Devel oping Countries. Stateof-the-Art Series, Nutrition Policy Discussion Paper n. 13. Geneva: Administrative Committee Coordination/Subcommitee on Nutrition, World Health Organization.

DIFRANCISCO, A.; HAKRABORTY, J.; CHOWDHURY, H. R.; YUNUS, M.; BAQUI, A. H.; SLDDIQUE, A. K. \& SACK, R. B., 1993. Acute toxicity of vitamin A given with vaccines in infancy. Lancet, 26:526527.

FAWZI, W. W.; CHALMERS, T. C.; HERRERA, M. G. \& MOSTELLER, F., 1993. Vitamin A supplementation and child mortality. A meta-analysis. JAMA, 269:898-903.

FLORENTINO, R.; TANCHOCO, C. C.; RAMOS, A. C.; MENDONZA, T. S.; NATIVIDAD, E. P.; TANGO, J. B. M. \& SOM MER, A., 1990. Tolerance of preschoolers to two dosage strengths of vitamin A preparation. American Journal of Clinical Nutrition, 52:694-700.

FLOYD, C. B. \& FREELING, P., 1992. Um estudo para estabelecer a incidência de efeitos adversos menores após a imunização de lactentes. In: Imunizações: Atualização (G. Oselka \& M. Yamamoto, org.), pp. 15-16, São Paulo: Secretaria de Estado da Saúde de São Paulo/Fundo das Nações Unidas para a Infância - UNICEF/Organização Panamericana da Saúde - OPS.

FAO/WHO (Food and Agriculture Organization/ World Health Organization), 1992. Final Report of the International Conference on Nutrition. Rome: $\mathrm{FAO} / \mathrm{WHO}$.

GLASZIOU, P. P. \& MacKERRRAS, D. E. M., 1993. Vitamin A supplementation in infectious diseases: A meta-analysis. BMJ, 306:366-370.

HATHCOCK, J. N.; HATTAN, D. G.; JENKINS, M. Y.; MCDONALD, J. T.; SUNDARESAN, P. R. \&WILKENING, V. L., 1990. Evaluation of vitamin A toxicity. American Journal of Clinical Nutrition, 52:183202.

IBGE (Fundação Instituto Brasileiro de Geografia e Estatística), 1991. Censo Demográfico - Bahia. Rio de Janeiro: IBGE.

JACOB, J. \& MANNINO, F., 1979. Increased intracranial pressure after diphtheria, tetanus, and pertussis immunization. American Journal of Diseases of Childhood, 133:217-218.
JONES, A. E.; JOHNS, A.; MAGRATH, D. I.; MELVILLESMITH, M. \& SHEFFIELD, F., 1992. Durabilidade da imunidade contra difteria, tétano e poliomielite após um esquema de imunização com três doses completado nos primeiros oito meses de vida. In: Imunizações: Atualização (G. Oselka \& M. Yamamoto, org.), p. 11, São Paulo: Secretaria de Estado da Saúde de São Paulo/Fundo das Nações Unidas para a Infância - UNICEF/Organização Panamericana da Saúde - OPS.

LONGHURST, R. \& TOMKINS, A., 1995. The role of care in nutrition - A neglected essential ingredient. WHO SCN News, 12:5.

PRADO, M. S.; ASSIS, A. M. O.; MARTINS, M. C.; NAZARÉ, M. P. A.; REZENDE, I. F. B. \& CONCEIÇÃO, M .E. P., 1995. Hipovitaminose A em crianças de áreas rurais do semi-árido baiano. Revista de Saúde Pública, 29:209-212.

RAY, S. K.; CHATTERJEE, R.; ASOK, M.; LAHIRI, S. K.; ROY, S. C. \& BANERJEE, S., 1984. A short report of untoward reactions following administration of D.P.T. vaccine and vitamin A in an under five. Indian Journal of Public Health, 28:213-216.

SANTOS, L. M. P.; ASSIS, A. M. O.; MARTINS, M. C.; ARAUJO, M. P. N.; MORRIS, S. M. \& BARRETO, M. L., 1996. Situação alimentar e nutricional de préescolares no semi-árido da Bahia: II - Hipovitaminose A. Revista de Saúde Pública, 30:67-74.

STABELL, C.; BALÉ, C.; SILVA, A. P.; OLSEN, J. \& AABY, P., 1995. No evidence of fontanelle-bulging episodes after vitamin A supplementation of 6 and 9-month-old infants in Guinea Bissau. European Journal of Clinical Nutrition, 49:73-74.

STRÖM, J., 1967. Further experience of reactions, especially of a cerebral nature, in conjunction with triple vaccination: A study based on vaccinations in Sweden 1959-65. BMJ, 4:320-323.

TONASCIA, J. A., 1992. Metaanalysis of published community trials: Impact of vitamin A on mortality. In: Bellagio Meeting on Vitmain A Deficiency and Childhood Mortality, Proceedings, p. 3. Bellagio: Rockefeller Foundation.

WEST, K. P.; KHATRY, S. K.; LECLERQ, S. C.; ADHIKARI, R.; SEE, L.; KATZ, J.; SHRESTHA, S. R.; PRADHAN, E. K.; POKHREL, R. P. \& SOM MER, A., 1992. Tolerance of young infants to a single, large dose of vitamin A: A randomized community trial in Nepal. Bulletin of the World Health Organization, 70: 733-739.

WHO/IVACG (World Health Organization/International Vitamin A Consultative Group), 1993. Using Immunization Contacts to Combat Vitamin A Deficiency, 1993. Report of an Informal Consultative Group of the World Health Organization. Geneva: WHO/IVACG. 